- Original Article

\title{
Association between Serum Uric Acid and Oxidative Stress in Korean Adults
}

\author{
Eun Jeong Ok, Kiyoung Kim, Sat Byul Park* \\ Department of Family Practice and Community Health, Ajou University School of Medicine, Suwon, Korea
}

Background: Oxidative stress is implicated in the pathogenesis and development of lifestyle-related diseases. In the present study, we evaluated the correlation between the serum uric acid (UA) levels and oxidative status in Korean adults.

Methods: The subjects were 5,093 individuals (2,041 women and 3,052 men) who underwent a health checkup between June 2012 and December 2016. Oxidative stress levels (derivatives of reactive oxygen metabolites [d-ROMs]) and antioxidant potential (biological antioxidant potential [BAP]) were measured. Metabolic markers, including UA, were also examined.

Results: Higher serum UA levels were associated with decreased levels of d-ROMs $(\mathrm{P}<0.05)$. The UA levels were positively associated with BAP levels $(\mathrm{P}<0.001)$.

Conclusion: Serum UA is related to oxidative status, especially antioxidant capacity, in Korean adults; UA may play a role in antioxidant defense systems in humans.

Keywords: Uric Acid; Oxidative Stress; Reactive Oxygen Species; Antioxidants

Received: March 3, 2017, Revised: August 28, 2017, Accepted: October 12, 2017

*Corresponding Author: Sat Byul Park https://orcid.org/0000-0003-1090-716X

Tel: +82-31-219-5325, Fax: +82-31-219-5218, E-mail: sbpark@ajou.ac.kr 


\section{INTRODUCTION}

In recent years, oxidative stress has been identified as an important factor in the pathogenesis and development of lifestyle-related diseases. Especially, it is well-known that oxidative stress causes the progression and acceleration of atherosclerosis. ${ }^{1,2)}$ Moreover, oxidative stress caused by the increased production of reactive oxygen species (ROS) and/or decreased effectiveness of the antioxidant system is implicated in the pathogenesis of various disease entities, such as arteriosclerosis, malignant tumors, and autoimmune diseases. ${ }^{3-6)}$

Several antioxidant defense systems present in humans may act to correct a cellular redox imbalance; these include uric acid (UA), bilirubin, and albumin. ${ }^{7-9)}$

A potent natural antioxidant, UA has been claimed to exert preventive effects against the development of age-related neurodegenerative diseases. ${ }^{10)}$ It protects against vascular damage mediated by oxidative stress following ischemia/reperfusion in rats. ${ }^{11)}$

In this study, we investigated the association between UA and the derivatives of reactive oxygen metabolites (d-ROMs), as an index of the products of ROS, and biological antioxidant potential (BAP), as an index of the antioxidant potential. ${ }^{1)}$

\section{METHODS}

\section{Subjects}

The subjects were 5,093 individuals (2,041 women and 3,052 men) who underwent a health checkup between June 2012 and December 2016. We excluded eight out of 5,101 people due to unavailability of data on serum UA. The majority of these subjects visited the clinic for regular health checkups, and therefore, did not have any serious health problems. The demographic information (age, sex, smoking, and drinking), medical history, and medication history of the subjects were investigated and recorded. Smoking status was categorized as non-smoking or currently smoking. Subjects who had been smoking cigarettes regularly 1 year before the survey were considered current smokers. Alcohol consumption was divided into the following categories: nondrinkers, current drinkers. The current drinker category was used for subjects who drank alcohol 2 times a week.

\section{Data Measurement}

Body mass index (BMI) was calculated as weight divided by height squared $\left(\mathrm{kg} / \mathrm{m}^{2}\right) .{ }^{12)}$ Blood pressure was measured by an automated sphygmomanometer after approximately 10 minutes of rest. Hypertension was defined as a systolic blood pressure (SBP) $\geq 140 \mathrm{~mm} \mathrm{Hg}$ or a diastolic blood pressure (DBP) $\geq 90 \mathrm{~mm} \mathrm{Hg}$ or if the participant was using antihypertensive medication. Diabetes was defined as the hemoglobin A1c level greater than $6.5 \%$ or use of hypoglycemic medications. Dyslipidemia was defined as a low-density lipoprotein (LDL) cholesterol level of $160 \mathrm{mg} / \mathrm{dL}$ or greater, or self-reported use of cholesterol-lowering supplements.

After overnight fasting, a venous blood sample was obtained in the morning. Each subject fasted for more than 10 hours before blood collection. Serum UA levels were assayed using a Hitachi Automatic Analyzer 7600 (Hitachi, Tokyo, Japan).

Both the oxidative stress level and antioxidant properties in serum were determined using an automated method. The oxidative stress level was measured via a d-ROM test (Diacron Srl, Grosseto, Italy), in which the amount of organic hydroperoxide converted into radicals that oxidize N,N-diethyl-p-phenylenediamine hydroperoxide was measured. ${ }^{3)}$ The results of the d-ROM test are expressed in an arbitrary unit called Carratelli unit (U.CARR). ${ }^{13-15)}$

In the BAP test (Diacron Srl), antioxidant potential was measured based on the capacity of the plasma sample to reduce ferric $\left(\mathrm{Fe} 3^{+}\right)$ ions to ferrous $\left(\mathrm{Fe} 2^{+}\right)$ions. ${ }^{1,16,17)}$ The results are expressed as $\mu \mathrm{mol} / \mathrm{L} .{ }^{13)}$

\section{Statistical Analysis}

The results of grouped data were expressed as the means \pm standard deviation (SD). Analysis of variance trend analysis was adapted. Distribution of d-ROMs and BAP values was left-skewed. Thus, a natural log-transformation was applied. We performed univariate analysis according to serum UA levels, and multivariate analysis after adjusting for age, sex, BMI, smoking status and alcohol drinking, SBP, total glyceride (TG), high-density lipoprotein (HDL), and LDL. Data analysis was conducted using IBM SPSS Statistics ver. 21.0 software (IBM Corp., Armonk, NY, USA). A P-value $<0.05$ was considered statistically significant.

\section{RESULTS}

\section{Baseline Characteristics}

Clinical and biochemical data (mean \pm SD, or subject numbers [percentage]) were categorized by serum UA values into three groups. Ranges of the 1st, 2nd, and 3rd tertile of serum UA values were 0.9-4.6, 4.7-5.9, and 6.0-11.6 mg/dL, respectively. The mean age of the subjects enrolled was $50.7 \pm 10.4$ years, and $59.9 \%(n=3,052)$ of them were men. The results showed that smoking and alcohol consumption was

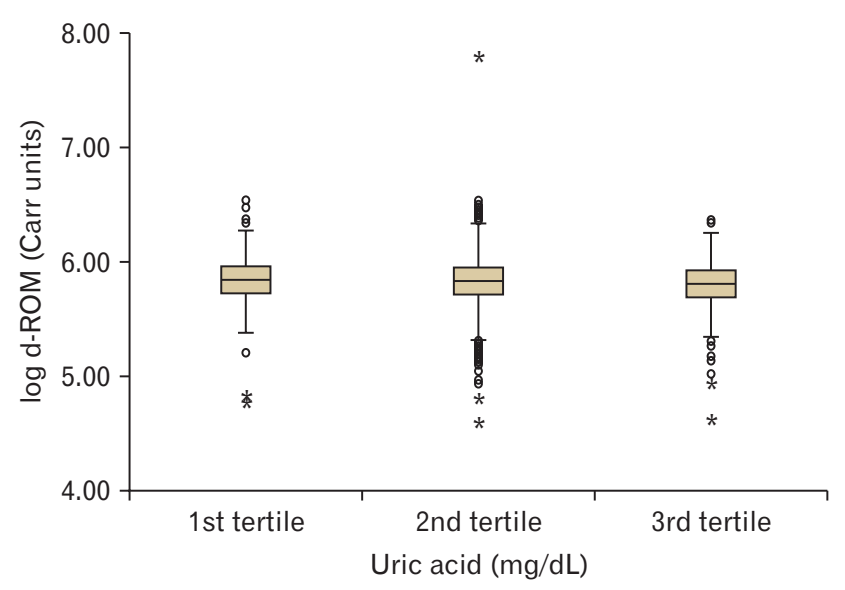

Figure 1. Relationship between d-ROMs and uric acid. d-ROM, derivatives of reactive oxygen metabolites. 
associated with increased serum UA levels. The mean d-ROM value was 346.6 \pm 1.2 U.CARR and BAP value was 2,181.6 $\pm 1.1 \mu \mathrm{mol} / \mathrm{L}$. Higher serum UA levels were associated with decreased levels of d-ROM (P for trend $<0.05$ ) and increased levels of BAP (P for trend $<0.001$ ) (Figures 1,2$)$.

On univariate analysis, serum UA levels were found to be positively correlated with age, BMI, and blood pressure. Clinical and laboratory characteristics of the subjects, according to their serum UA levels, are listed in Table 1.

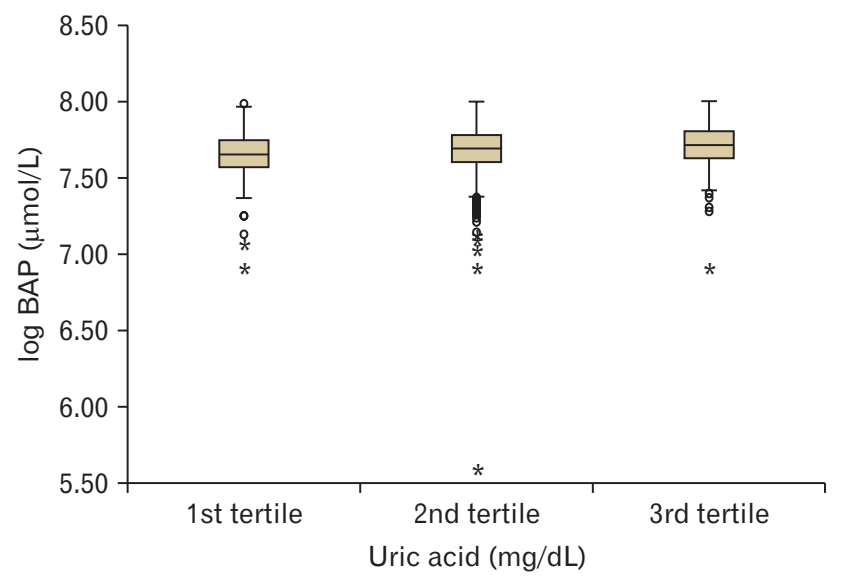

Figure 2. Relationship between BAP and uric acid. BAP, biological antioxidant potential.

\section{Relationships of Derivatives of Reactive Oxygen Metabolites and Biological Antioxidant Potential with Uric Acid}

There were significant differences according to serum UA levels. These associations remained statistically significant after adjusting for age, sex, BMI, smoking status and alcohol consumption, SBP, TG, HDL, and LDL (Table 2).

\section{DISCUSSION}

This study shows that higher tertiles of serum UA levels were related to decreased levels of d-ROM and increased levels of BAP in subjects. Multiple regression analysis showed that the levels of d-ROM and BAP

Table 2. OR between uric acid levels and d-ROM or BAP

\begin{tabular}{|c|c|c|c|c|c|}
\hline \multirow{2}{*}{ Variable } & \multirow{2}{*}{$\begin{array}{l}\text { Range } \\
\text { (mg/dL) }\end{array}$} & \multicolumn{2}{|c|}{ Univariate analysis } & \multicolumn{2}{|c|}{ Multivariate analysis } \\
\hline & & $\mathrm{OR}$ & P-value & $\mathrm{OR}$ & P-value \\
\hline \multicolumn{6}{|l|}{ D-ROM } \\
\hline 1st tertile & $0.9-4.6$ & 1 & 0.035 & 1 & 0.007 \\
\hline 2nd tertile & $4.7-5.9$ & 0.907 & 0.405 & 1.457 & 0.008 \\
\hline 3rd tertile & $6.0-11.6$ & 0.742 & 0.030 & 1.795 & 0.002 \\
\hline \multicolumn{6}{|l|}{ BAP } \\
\hline 1st tertile & $0.9-4.6$ & 1 & $<0.001$ & 1 & 0.006 \\
\hline 2nd tertile & $4.7-5.9$ & 1.670 & $<0.001$ & 1.489 & 0.004 \\
\hline 3rd tertile & $6.0-11.6$ & 2.169 & $<0.001$ & 1.759 & 0.002 \\
\hline
\end{tabular}

Multivariate analysis was performed after adjusting for age, sex, body mass index, smoking status and alcohol consumption, systolic blood pressure, total glyceride, high-density lipoprotein, and low-density lipoprotein using binary logistic regression analysis.

$\mathrm{OR}$, odds ratio; d-ROM, derivatives of reactive oxygen metabolites; BAP, biological antioxidant potential.

Table 1. Demographic and clinical characteristics of subjects, according to serum uric acid levels

\begin{tabular}{|c|c|c|c|c|c|}
\hline \multirow{2}{*}{ Characteristic } & \multicolumn{4}{|c|}{ UA grading* } & \multirow{2}{*}{ P-value ${ }^{\dagger}$} \\
\hline & Total $(\mathrm{N}=5,093)$ & $\mathrm{T} 1(\mathrm{n}=1,755)$ & T2 $(n=1,628)$ & T3 $(n=1,710)$ & \\
\hline Uric acid (mg/dL) & & $3.8 \pm 0.6(0.9-4.6)$ & $5.3 \pm 0.4(4.7-5.9)$ & $7.0 \pm 0.9(6.0-11.6)$ & \\
\hline Age (y) & $50.7 \pm 10.4$ & $50.8 \pm 10.6$ & $51.6 \pm 10.2$ & $49.7 \pm 10.3$ & $<0.001$ \\
\hline Male & $3,052(59.9)$ & $412(23.5)$ & $1,038(63.8)$ & $1,602(93.7)$ & $<0.001$ \\
\hline Body mass index $\left(\mathrm{kg} / \mathrm{m}^{2}\right)$ & $24.0 \pm 3.2$ & $22.8 \pm 3.0$ & $23.8 \pm 2.9$ & $25.2 \pm 3.0$ & $<0.001$ \\
\hline Systolic blood pressure (mm Hg) & $118 \pm 14$ & $116 \pm 15$ & $118 \pm 15$ & $121 \pm 14$ & $<0.001$ \\
\hline Diastolic blood pressure (mm Hg) & $75 \pm 11$ & $73 \pm 10$ & $75 \pm 11$ & $78 \pm 10$ & $<0.001$ \\
\hline Total glyceride (mg/dL) & $122.8 \pm 88.0$ & $99.2 \pm 64.0$ & $120.4 \pm 84.9$ & $149.3 \pm 103.7$ & $<0.001$ \\
\hline High-density lipoprotein cholesterol (mg/dL) & $55.4 \pm 14.5$ & $60.2 \pm 14.6$ & $55.6 \pm 14.8$ & $50.2 \pm 12.2$ & $<0.001$ \\
\hline Low-density lipoprotein cholesterol (mg/dL) & $122.7 \pm 34.3$ & $119.3 \pm 32.8$ & $123.6 \pm 34.2$ & $125.7 \pm 35.5$ & $<0.001$ \\
\hline Fasting plasma glucose (mg/dL) & $98.3 \pm 20.5$ & $98.0 \pm 24.3$ & $98.1 \pm 19.8$ & $98.8 \pm 16.6$ & 0.560 \\
\hline Hemoglobin A1c (\%) & $5.6 \pm 0.8$ & $5.9 \pm 1.0$ & $5.7 \pm 0.7$ & $5.7 \pm 0.7$ & 0.266 \\
\hline Diabetes mellitus & $326(6.4)$ & $135(7.7)$ & $109(6.7)$ & $82(4.8)$ & 0.093 \\
\hline Hypertention & $1,119(22.0)$ & $300(17.1)$ & $377(23.2)$ & $442(25.8)$ & 0.001 \\
\hline Dyslipidemia & $951(18.7)$ & $281(16.0)$ & $329(20.2)$ & $341(19.9)$ & 0.001 \\
\hline Current smokers & $995(19.5)$ & $146(8.3)$ & $334(20.5)$ & $515(30.1)$ & $<0.001$ \\
\hline Drinking alcohol & $2,813(55)$ & $683(38.9)$ & $911(56.0)$ & $1,219(71.3)$ & $<0.001$ \\
\hline Derivatives of reactive oxygen metabolites (Carr units) & $346.6 \pm 1.2$ & $352.3 \pm 1.2$ & $346.2 \pm 1.2$ & $338.5 \pm 1.2$ & 0.009 \\
\hline Biological antioxidant potential $(\mu \mathrm{M})$ & $2,181.6 \pm 1.1$ & $2,107.0 \pm 1.1$ & $2,178.7 \pm 1.1$ & $2,239.7 \pm 1.1$ & $<0.001$ \\
\hline
\end{tabular}

Values are presented as mean \pm standard deviation (range), mean \pm standard deviation, or number (\%).

${ }^{*} \mathrm{~T} 1$, 1st tertile; $\mathrm{T} 2$, 2nd tertile; $\mathrm{T} 3$, 3rd tertile. ${ }^{\dagger}$ One-way analysis of variance, $\mathrm{P}<0.05$. 
were closely related to serum UA levels, independently of age, sex, BMI, blood pressure, LDL cholesterol, HDL cholesterol, triglyceride, smoking, and alcohol consumption. These findings suggest a positive correlation between the levels of serum UA and BAP.

Previous studies have demonstrated that circulating UA may exert either anti- or pro-oxidant activity in-vivo, ${ }^{18,19)}$ but anti- and pro-oxidant markers have not previously been simultaneously measured in a large population and their relation to circulating UA levels has not been assessed, as in the present study. We demonstrate that serum UA has antioxidant capacity.

Fukui et al. ${ }^{1)}$ showed that UA was positively associated with BAP. As a factor that influences BAP, the serum UA level was selected; UA is recognized as the primary defense against oxidative stress in extracellular fluids. ${ }^{9)}$ On the other hand, A study conducted in Japan showed that higher serum UA levels were associated with increased levels of $d$ ROMs in both sexes. ${ }^{7)}$ The difference in some observations might be attributed to variations in sample size, the statistical methods used, and the consideration or non-consideration of albuminuric status. ${ }^{7)}$

It is well-known that high plasma UA level is strongly associated with peripheral, carotid, and coronary vascular diseases; development of stroke; and vascular dementia. ${ }^{9,17,20,21)}$ The relationship of UA with cardiovascular events is particularly strong, especially in patients at high risk for heart diseases. Whether UA has a causal relationship in these conditions remains to be determined.

Oxidative stress may be associated with not only lifestyle-related diseases but also various other diseases. Therefore, our results suggest the significance of oxidative stress measurement in a large number of subjects. For a better understanding of these double-sided actions of UA, future studies should clarify whether the harmful effects of UA are caused by its pro-oxidant properties, and conversely, whether the beneficial effects of UA are attributable to its antioxidant potential.

A limitation of our study is that we analyzed data from participants who voluntarily visited a health promotion center; this group might not be representative of the general population.

In conclusion, in the present cross-sectional study, serum UA level was found to be closely associated with d-ROM and BAP levels in Korean adults. Serum UA levels are related to oxidative status, especially antioxidant capacity. Therefore, UA may play a role in the antioxidant defense systems in humans.

\section{CONFLICT OF INTEREST}

No potential conflict of interest relevant to this article was reported.

\section{REFERENCES}

1. Fukui T, Yamauchi K, Maruyama M, Yasuda T, Kohno M, Abe Y. Significance of measuring oxidative stress in lifestyle-related diseases from the viewpoint of correlation between d-ROMs and BAP in Japanese subjects. Hypertens Res 2011;34:1041-5.

2. Aviram M. Review of human studies on oxidative damage and antioxi- dant protection related to cardiovascular diseases. Free Radic Res 2000;33 Suppl:S85-97.

3. Kamezaki F, Yamashita K, Kubara T, Suzuki Y, Tanaka S, Rkouzuma R, et al. Derivatives of reactive oxygen metabolites correlates with highsensitivity C-reactive protein. J Atheroscler Thromb 2008;15:206-12.

4. Katsabeki-Katsafli A, Kerenidi T, Kostikas K, Dalaveris E, Kiropoulos TS, Gogou E, et al. Serum vascular endothelial growth factor is related to systemic oxidative stress in patients with lung cancer. Lung Cancer 2008;60:271-6.

5. Kotani K, Koibuchi H, Miyamoto M, Yamada T, Taniguchi N. Relationship between reactive oxygen metabolites and carotid intima-media thickness in subjects with hypercholesterolemia. Med Princ Pract 2010;19:496-8.

6. Sakane N, Fujiwara S, Sano Y, Domichi M, Tsuzaki K, Matsuoka Y, et al. Oxidative stress, inflammation, and atherosclerotic changes in retinal arteries in the Japanese population; results from the Mima study. Endocr J 2008;55:485-8.

7. Ishizaka Y, Yamakado M, Toda A, Tani M, Ishizaka N. Relationship between serum uric acid and serum oxidative stress markers in the Japanese general population. Nephron Clin Pract 2014;128:49-56.

8. Stocker R, Yamamoto Y, McDonagh AF, Glazer AN, Ames BN. Bilirubin is an antioxidant of possible physiological importance. Science 1987; 235:1043-6.

9. Ihara H, Hashizume N, Hasegawa T, Yoshida M. Antioxidant capacities of ascorbic acid, uric acid, alpha-tocopherol, and bilirubin can be measured in the presence of another antioxidant, serum albumin. J Clin Lab Anal 2004;18:45-9.

10. Cutler RG, Camandola S, Malott KF, Edelhauser MA, Mattson MP. The role of uric acid and methyl derivatives in the prevention of age-related neurodegenerative disorders. Curr Top Med Chem 2015;15:2233-8.

11. Onetti Y, Dantas AP, Perez B, Cugota R, Chamorro A, Planas AM, et al. Middle cerebral artery remodeling following transient brain ischemia is linked to early postischemic hyperemia: a target of uric acid treatment. Am J Physiol Heart Circ Physiol 2015;308:H862-74.

12. National Institutes of Health. Clinical guidelines on the identification, evaluation, and treatment of overweight and obesity in adults: the evidence report. Obes Res 1998;6 Suppl 2:51S-209S.

13. Pasquini A, Luchetti E, Marchetti V, Cardini G, Iorio EL. Analytical performances of d-ROMs test and BAP test in canine plasma: definition of the normal range in healthy Labrador dogs. Vet Res Commun 2008;32:137-43.

14. Cesarone MR, Belcaro G, Carratelli M, Cornelli U, De Sanctis MT, Incandela L, et al. A simple test to monitor oxidative stress. Int Angiol 1999;18:127-30.

15. Trotti R, Carratelli M, Barbieri M. Performance and clinical application of a new, fast method for the detection of hydroperoxides in serum. Panminerva Med 2002;44:37-40.

16. Dohi K, Satoh K, Ohtaki H, Shioda S, Miyake Y, Shindo M, et al. Elevated plasma levels of bilirubin in patients with neurotrauma reflect its pathophysiological role in free radical scavenging. In Vivo 2005;19: 855-60.

17. Moriarity JT, Folsom AR, Iribarren C, Nieto FJ, Rosamond WD. Serum uric acid and risk of coronary heart disease: Atherosclerosis Risk in Communities (ARIC) Study. Ann Epidemiol 2000;10:136-43.

18. George J, Carr E, Davies J, Belch JJ, Struthers A. High-dose allopurinol 
improves endothelial function by profoundly reducing vascular oxidative stress and not by lowering uric acid. Circulation 2006;114:2508-16.

19. Fabbrini E, Serafini M, Colic Baric I, Hazen SL, Klein S. Effect of plasma uric acid on antioxidant capacity, oxidative stress, and insulin sensitivity in obese subjects. Diabetes 2014;63:976-81.

20. Schretlen DJ, Inscore AB, Vannorsdall TD, Kraut M, Pearlson GD, Gor- don B, et al. Serum uric acid and brain ischemia in normal elderly adults. Neurology 2007;69:1418-23.

21. Bos MJ, Koudstaal PJ, Hofman A, Witteman JC, Breteler MM. Uric acid is a risk factor for myocardial infarction and stroke: the Rotterdam study. Stroke 2006;37:1503-7. 